LETTER TO EDITOR

\title{
Glucose Tolerance Test in Clinical Practice is Varied in Basic Research
}

\author{
Kai-Chun Cheng ${ }^{1}$, Yingxiao $L^{1,2}$ and Juei-Tang Cheng ${ }^{2,3^{*}}$ \\ ${ }^{1}$ Department of Psychosomatic Internal Medicine, Graduate School of Medical and Dental Sciences, \\ Kagoshima University, Kagoshima, Japan \\ ${ }^{2}$ Department of Medical Research, Chi-Mei Medical Center, Yong Kang, Tainan City, Taiwan \\ ${ }^{3}$ Institute of Medical Science, College of Health Science, Chang Jung Christian University, Gueiren, Tainan \\ City, Taiwan
}

*Corresponding author: Professor Juei-Tang Cheng, Institute of Medical Sciences, Chang Jung Christian University, Gueiren, Tainan City, 71101, Taiwan, Tel: +886-6-251-7864, Fax: +886-6-283-2639, E-mail: jtcheng@mail.cjcu.edu.tw

In diabetic patients, blood glucose levels often elevate markedly after a meal, mainly associated with the progress of diabetic complications [1]. They belong to a category of hyperglycemia which is abnormal but is still not reach the criteria of diabetes mellitus (DM). Generally, they are classified as the impaired glucose tolerance [2]. In clinics, oral glucose tolerance test (OGTT) is widely used to diagnostic for the impaired glucose tolerance (IGT) and/or type-2 DM (T2DM) while IGT is obtained from a 2-hour post-challenge plasma glucose level about 140-200 mg/dl during OGTT [2]. In recent, the shape of glucose curve during OGTT has been pointed out to reveal the risk of developing IGT $[3,4]$, which is also associated with variations in insulin sensitivity and secretion in youth $[4,5]$. Moreover, the patterns of insulin concentration during an OGTT have been used to predict the development of T2DM from a Japanese Americans study for 10-11 years [6]. Recently, using a multiple sample in 2-hour OGTT, the variation in time showing glucose peak has also been suggested as an indicator of prediabetes and/or $\beta$-cell function better than glucose curve shape [7].

Moreover, using the robust methodology, a study in obese adolescents found that the shape of glucose response curve during OGTT, monophasic versus biphasic, identifies the variations in insulin secretion and sensitivity [4]. The monophasic group had a marked higher glucose, insulin, c-peptide and free fatty acid OGTT areas under the curve (AUC) than the biphasic group, but no differences in the levels of glucagon, total glu- cagon-like peptide 1 (GLP-1), glucose-dependent insulinotropic polypeptide (GIP), and pancreatic polypeptide. Also, the monophasic group showed lower in vivo hepatic and peripheral insulin sensitivity, lack of compensatory insulin secretion, and impaired $\beta$-cell function related to insulin sensitivity that may let them at a high risk of developing T2DM [5]. Additionally, another study [8] used the same method to investigate the dynamic response of $\beta$ cells, independently of the insulin sensitivity [9]. Also, the volunteers who kept monophasic shape only or switched to monophasic from biphasic shape over time had a higher risk of impaired glucose metabolism while biphasic shape had a lower risk [8]. Similarly, the risk of T2DM was double in monophasic shape compared to the group with biphasic shape [3]. However, the peripheral insulin sensitivity was similar between two groups although monophasic shape showed a higher insulin output but a less $\beta$-cell glucose sensitivity [8]. Therefore, OGTT has also been developed to investigate the pathophysiology of diabetes. However, the peak and decay in plasma glucose levels during the OGTT reflect the interplay between several factors, including the glucose intestinal absorption rate, insulin sensitivity, $\beta$-cell function and components, such as $\beta$-cell glucose sensitivity, $\beta$-cell insulin sensitivity rate and enhanced factor, in addition to the secretion of gut hormones. Thus, application of OGTT in the diagnosis of impaired glucose tolerance is useful but it seems limited in the study of pathogenesis of T2DM without another indicator(s). 
Recently, along with the recent pandemics of obesity worldwide, the prevalence of not only T2DM but also nonalcoholic fatty liver disease (NAFLD) is rapidly increasing worldwide. Therefore, recently, the association and/or relevance of T2DM, NAFLD, and glucose and insulin metabolism have been widely linked using OGTT in clinics. The postprandial insulin secretion pattern is associated with histological severity in non-alcoholic fatty liver disease (NAFLD) patients without prior known T2DM [10]. Recently, the risk of transient postprandial hypoglycemia in NAFLD has been identified using OGTT [11]. Also, the value of a 1-hour OGTT glucose $\geq 155 \mathrm{mg} /$ $\mathrm{dL}$ has been suggested as helpful to identify a subset of non-glucose tolerant individuals at risk for NAFLD [12]. Moreover, Non-Alcoholic Steatohepatitis (NASH) has also been linked with T2DM, particularly a high prevalence of NASH (56.49\%) in patients with T2DM with duration of more than 5 Years [13]. Therefore, a new application of OGTT for diagnosis of NAFLD or NASH without overt T2DM is suggested in clinical practice.

In basic research, OGTT is mainly used to investigate the glucose homeostasis in animals. Insulin resistance (IR) or insulin sensitivity has ever been characterized in animals using the data from glucose-insulin index that was also calculated from OGTT [14]. But it was recently replaced by a simple method named HOME-IR [15], although it's application has ever been challenged [16]. Additionally, OGTT has also been used as a model to mimic the postprandial state of hyperglycemia as well as provides information on the glucose-lowering effect as a consequence of changes in glucose utilization [17]. Basically, OGTT or meal tolerance test mimics the glucose and insulin dynamics under physiological conditions more closely than conditions of the glucose clamp or insulin sensitivity test. The postprandial rise in plasma insulin enables appropriate disposal of blood glucose in the absorptive state, while the fall in plasma insulin contributes to maintaining glucose homeostasis in the post-absorptive state and during the starvation. Therefore, it is not suitable to ignore the blood insulin levels that are dependent on the ability of pancreatic beta-celIs in response to the challenges from plasma glucose levels. Moreover, determinations are easily influenced by a number of physiological and environmental factors in addition to pathological situations. Therefore, adequate experience of the tests is important to reduce the bias in animals both before and during the assays.

Impaired glucose tolerance is mostly reflected in a larger incremental area under the curve (AUC) of the plasma glucose disappearance curve during OGTT. Results in OGTT showed a marked increase in $A U C_{0-120 \text { min }}$ from the diabetic group indicating the success of diabetic model. Similarly, glucose tolerance is also significantly changed in the mutant mice compared with their wild-type littermates [18]. After the treatment with testing substance, either herbal extract or nutrient, the slope of the glucose disposal phase is markedly incre- ased and AUC is significantly decreased comparing to the vehicle-treated group as control. It means that the testing substance has an ability to alleviate the impaired glucose tolerance. However, OGTT mimics the glucose and insulin dynamics; it is still not enough to conclude that the testing substance enhanced glucose utilization as the mechanistic results because the $\alpha$-glucosidase inhibitor-like action may result in same changes [19]. Therefore, more studies are usually required such as insulin-tolerance test, glucose uptake, glycolysis and others [18]. It means that data showing a marked reduction in AUC only from OGTT are not enough to support the conclusion regarding an increase in glucose utilization as the action mechanism for testing substance. However, it has been applied in some basic studies regardless the glucose and insulin integrations.

In the integrated model [9], glucose is introduced as a two-compartment disposition model under both insulin dependent and insulin-independent elimination from one central compartment. Insulin is mentioned as a one-compartment model with linear elimination while insulin secretion is separated into first and second-phase secretion [20]. The first-phase secretion is shown as a bolus dose which enters into the plasma compartment. The second-phase secretion is parameterized to be equal to elimination at the baseline and to raise with the increased glucose concentrations, similar to glucose production. However, the main limitation of this model is that it has been developed based on the data covering only a short time that may prohibit the use in analysis of drug with slowly developing effects, particularly the drugs acting on gene transcription. Therefore, this model is not widely applied although it seems useful to analyze the drug effects [21].

In conclusion, glucose tolerance test used in clinic is extremely varied in basic research. Diagnosis of diseases including diabetes and/or hepatic disorders is the main aim in clinical practice while the impaired glucose tolerance in animals is mainly targeted in basic studies. However, reduction in AUC only from animals received OGTT is still not enough to conclude the action mechanism via increase in glucose utilization.

\section{References}

1. Ceriello A (2005) Postprandial hyperglycemia and diabetes complications: Is it time to treat? Diabetes 54: 1-7.

2. Adam JM, Josten D (2008) Isolated post-challenge hyperglycemia: Concept and clinical significance. Acta Med Indones 40: 171-175.

3. Abdul-Ghani MA, Lyssenko V, Tuomi T, Defronzo RA, Groop $L$ (2010) The shape of plasma glucose concentration curve during OGTT predicts future risk of type 2 diabetes. Diabetes Metab Res Rev 26: 280-286.

4. Kim JY, Michaliszyn SF, Nasr A, Lee S, Tfayli $\mathrm{H}$, et al. (2016) The shape of the glucose response curve during an oral glucose tolerance test heralds biomarkers of type 2 diabetes risk in obese youth. Diabetes Care 39: 1431-1439. 
5. Nolfe G, Spreghini MR, Sforza RW, Morino G, Manco M (2012) Beyond the morphology of the glucose curve following an oral glucose tolerance test in obese youth. Eur $\mathrm{J}$ Endocrinol 166: 107-114.

6. Hayashi T, Boyko EJ, Sato KK, McNeely MJ, Leonetti DL, et al. (2013) Patterns of insulin concentration during the OGTT predict the risk of type 2 diabetes in Japanese Americans. Diabetes Care 36: 1229-1235.

7. Chung ST, Ha J, Onuzuruike AU, Kasturi K, Galvan-De La Cruz M, et al. (2017) Time to glucose peak during an oral glucose tolerance test identifies prediabetes risk. Clin Endocrinol (Oxf) 87: 484-491.

8. Manco M, Nolfe G, Pataky Z, Monti L, Porcellati F, et al. (2017) Shape of the OGTT glucose curve and risk of impaired glucose metabolism in the EGIR-RISC cohort. Metabolism 70: 42-50.

9. Silber HE, Jauslin PM, Frey N, Karlsson MO (2010) An integrated model for the glucose-insulin system. Basic Clin Pharmacol Toxicol 106: 189-194.

10. Kimura $Y$, Hyogo H, Ishitobi T, Nabeshima $Y$, Arihiro $K$, et al. (2011) Postprandial insulin secretion pattern is associated with histological severity in non-alcoholic fatty liver disease patients without prior known diabetes mellitus. $\mathrm{J}$ Gastroenterol Hepatol 26: 517-522.

11. Morio R, Hyogo H, Hatooka M, Morio K, Kan H, et al. (2017) The risk of transient postprandial oxyhypoglycemia in nonalcoholic fatty liver disease. J Gastroenterol 52: 253-262.

12. Sesti G, Hribal ML, Fiorentino TV, Sciacqua A, Perticone F (2014) Elevated $1 \mathrm{~h}$ postload plasma glucose levels identify adults with normal glucose tolerance but increased risk of non-alcoholic fatty liver disease. BMJ Open Diabetes Res Care 2: e000016.
13. Seetlani NK, Memon AR, Tanveer S, Ali A, Ali P, et al. (2016) Frequency of non-alcoholic steatohepatitis on histopathology in patients of type 2 diabetes mellitus with duration of more than 5 years. J Coll Physicians Surg Pak 26: 643-646.

14. Peth JA, Kinnick TR, Youngblood EB, Tritschler HJ, Henriksen EJ (2000) Effects of a unique conjugate of alpha-lipoic acid and gamma-linolenic acid on insulin action in obese Zucker rats. Am J Physiol Regul Integr Comp Physiol 278: 453-459.

15. Matthews DR, Hosker JP, Rudenski AS, Naylor BA, Treacher DF, et al. (1985) Homeostasis model assessment: Insulin resistance and beta-cell function from fasting plasma glucose and insulin concentrations in man. Diabetologia 28: 412-419.

16. Wallace TM, Levy JC, Matthews DR (2004) Use and abuse of HOMA modeling. Diabetes Care 27: 1487-1495.

17. Lefebvre PJ, Scheen AJ (1998) The postprandial state and risk of cardiovascular disease. Diabet Med 15: S63-S68.

18. Chen A, Brar B, Choi CS, Rousso D, Vaughan J, et al. (2006) Urocortin 2 modulates glucose utilization and insulin sensitivity in skeletal muscle. Proc Natl Acad Sci U S A 103: 16580-16585.

19. Priya CL, Bhaskara Rao KV (2016) Postprandial Antihyperglycemic and antioxidant activities of acalypha indica linn stem extract: An in-vivo study. Pharmacogn Mag 12: S475-S481.

20. Silber HE, Jauslin PM, Frey N, Gieschke R, Simonsson US, et al. (2007) An integrated model for glucose and insulin regulation in healthy volunteers and type 2 diabetic patients following intravenous glucose provocations. J Clin Pharmacol 47: 1159-1171.

21. Ferrannini $E$, Mari A (2014) $\beta$-Cell function in type 2 diabetes. Metabolism 63: 1217-1227. 